\title{
Aproximações acadêmicas sobre a temática violência contra a mulher na perspectiva do futuro profissional enfermeiro
}

\author{
Academic approaches about the theme of violence against women from the perspective of \\ the future professional nurse
}
Enfoques académicos sobre el tema de la violencia contra la mujer desde la perspectiva del futuro profesional de enfermería

Edinéia Celestino Coutinho Fernandes ${ }^{1 *}$, Carlos Augusto de Oliveira Panta ${ }^{2}$, Marcela Milrea Araújo Barros ${ }^{3}$.

\section{RESUMO}

Objetivo: Descrever as abordagens no ensino sobre a temática violência contra mulher na perspectiva dos acadêmicos e futuros profissionais de enfermagem. Métodos: Estudo com enfoque investigatório-descritivo, sustentado pela abordagem qualitativa realizado em uma Instituição de Ensino Superior no Norte do Brasil. A análise de dados foi realizada a partir da análise de conteúdo. Resultados: Participaram do estudo 22 acadêmicos de enfermagem do oitavo ao décimo período com predominância do sexo feminino. As práticas educativas referidas são as rodas de conversa, aulas teóricas e palestras. As temáticas violência obstétrica, acolhimento, redes sociais de apoio e abordagem à mulher são as mais discutidas. Como futuro profissional, o acadêmico percebe a necessidade de aprofundamento de conhecimento específico, para uma abordagem qualificada, sobretudo no campo prático. Conclusão: $A$ temática necessita de maior aprofundamento ainda na graduação em enfermagem, com uso de metodologias ativas e estratégias que fortaleçam a integração ensino e serviço, que potencializem de maneira crítica e problematizadora as habilidades e competências do futuro profissional. Estabelece-se à importância da realização de novos estudos voltados para abordagens que relacionem formação acadêmica e prática profissional em enfermagem a fim de contribuir com uma assistência humanizada, protetiva e eficaz à essa mulher.

Palavras-chave: Violência, Mulher, Enfermagem.

\begin{abstract}
Objective: To describe teaching approaches on the theme of violence against women from the perspective of academics and future nursing professionals. Methods: Study with an investigative-descriptive focus, supported by a qualitative approach carried out in a Higher Education Institution in Northern Brazil. Data analysis was performed from content analysis. Results: Twenty-two nursing students from the eighth to the tenth period, predominantly female, participated in the study. The educational practices referred to are conversation circles, theoretical classes and lectures. Thematic obstetric violence, reception, social support networks and approach to women are the most discussed. As a future professional, the academic perceives the need to deepen specific knowledge for a qualified approach, especially in the practical field. Conclusion: The theme still needs further deepening in nursing graduation, with the use of active methodologies and strategies that strengthen the teaching and service integration, which critically and problematize the skills and competences of the future professional. The importance of carrying out new studies aimed at approaches that relate academic training and professional practice in nursing is established in order to contribute to humanized, protective and effective care for this woman.
\end{abstract}

Keywords: Violence, Women, Nursing.

\footnotetext{
${ }^{1}$ Faculdade Interamericana de Porto Velho - UNIRON, Porto Velho - RO.

*E-mail: edineiacoutinho53@gmail.com
} 


\section{RESUMEN}

Objetivo: Describir enfoques de enseñanza sobre el tema de la violencia contra la mujer desde la perspectiva de académicos y futuros profesionales de enfermería. Métodos: Estudio con enfoque investigativo-descriptivo, apoyado en un abordaje cualitativo realizado en una Institución de Educación Superior en el Norte de Brasil. El análisis de datos se realizó a partir del análisis de contenido. Resultados: Participaron del estudio veintidós estudiantes de enfermería del octavo al décimo período, predominantemente mujeres. Las prácticas educativas a las que se hace referencia son círculos de conversación, clases teóricas y conferencias. La temática violencia obstétrica, acogida, redes de apoyo social y acercamiento a la mujer son las más discutidas. Como futuro profesional, el académico percibe la necesidad de profundizar conocimientos específicos para un enfoque calificado, especialmente en el campo práctico. Conclusión: El tema aún necesita profundizar más en la graduación de enfermería, con el uso de metodologías y estrategias activas que fortalezcan la integración docente y de servicios, que critiquen y problematicen las habilidades y competencias del futuro profesional. Se establece la importancia de realizar nuevos estudios orientados a enfoques que relacionen la formación académica y la práctica profesional en enfermería con el fin de contribuir al cuidado humanizado, protector y eficaz de esta mujer.

Palabras clave: Violencia, Mujeres, Enfermería.

\section{INTRODUÇÃO}

A violência é um obstáculo social que se processa quando um agressor inicia um comportamento hostil em relação à vítima. De tal forma, se enquadra como uma relação de dominância, em que a vítima não pode se defender sujeitando-se a diversos abusos (ROSÁRIO AC, et al., 2017). De acordo com a Organização Mundial de Saúde (OMS) (2018), a violência de uma forma geral, é o uso de força física, poder na forma de ameaça, consigo próprio, demais indivíduos, entre grupos ou comunidades que possam resultar em sofrimento, óbito, dano psicológico, agravamento no desenvolvimento ou privação.

A violência contra a mulher (VCM) é uma das principais condutas de violação dos direitos humanos das mulheres, que consequentemente este fenômeno afetará esse gênero em diferentes classes sociais, origens, idades, religiões, estado civil, educação, raça e até mesmo orientação sexual. Pode ser implementado de diferentes formas (familiar, mental, física, moral, hereditária, sexual, tráfico de mulheres, assédio sexual, etc.) (SILVA VG e RIBEIRO PM, 2020).

A violência contra a mulher é um fenômeno mundial, como um problema de saúde pública, mediante os inúmeros agravos gerados à sua saúde e riscos de feminicídio (TEIXEIRA MCB, et al., 2018). O conhecimento acerca dessa temática e as condutas adotadas pelos acadêmicos de enfermagem, necessitam ser orientadas durante a graduação para melhor condução e enfrentamento desse problema com intervenções oportunas (DELZIOVO CR, et al., 2018). Na maioria das vezes, por não ser abordada durante a formação, provavelmente resultará em fragilidade para o futuro enfermeiro no cuidado às mulheres que se encontram nesse tipo de vulnerabilidade. Limitações como essas, constituem entraves para uma assistência integral (XAVIER AAP e SILVA EG, 2019).

Considerando as Diretrizes Curriculares Nacionais (DCN) para o curso de graduação em Enfermagem e a realidade dos agravos que acometem a população feminina a partir do seu contexto histórico, social e político, entende-se que a formação dos acadêmicos de enfermagem necessita ser redesenhada nos programas curriculares atuais (BRASIL, 2016).

Do ponto de vista acadêmico, se propõe a desvelar dados relevantes acerca do olhar do acadêmico de enfermagem frente à mulher vítima de violência, enquanto futuros profissionais que atuarão no acolhimento, enfrentamento e atendimento as mulheres que sofrem com a violência. Nesta perspectiva, surge a questão norteadora: Quais as aproximações acadêmicas sobre a temática violência contra mulher no curso de enfermagem? O estudo tem por objetivo descrever as abordagens no ensino sobre a temática violência contra mulher na perspectiva dos acadêmicos e futuros profissionais de enfermagem no Norte do Brasil. 


\section{MÉTODOS}

Trata-se de uma pesquisa de campo com escopo investigatório e descritivo de abordagem qualitativa. $O$ cenário do estudo foi uma Instituição de Ensino Superior (IES) na região Norte do Brasil. Foram estabelecidos como critérios de inclusão: acadêmicos (as) do curso de enfermagem, matriculados do oitavo ao décimo período, que já cursaram a disciplina Assistência Integral a Saúde da Mulher ou disciplina com grade curricular similar e que aceitaram participar voluntariamente do estudo, assinando o Termo de Consentimento Livre Esclarecido (TCLE). Foram excluídos da pesquisa os acadêmicos de enfermagem que não estavam presentes no período selecionado para coleta de dados.

A aplicação do questionário ocorreu nas dependências da instituição, nos intervalos das aulas práticas, com abordagem individualizada, distanciamento estabelecido pelas normas institucionais e uso de equipamentos de proteção individual, em consideração a situação de pandemia da Covid-19, conforme disponibilidade do acadêmico em momento reservado, garantido o sigilo e a privacidade na sua participação voluntária. $\mathrm{O}$ instrumento foi preenchido e recolhido na sequência e o tempo aproximado de resposta ao questionário foi de 20 a 30 minutos. Os dados foram analisados a partir da análise de conteúdo.

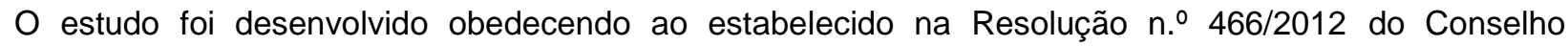
Nacional de Saúde/Ministério da Saúde. Por se tratar de pesquisa com seres humanos, o estudo foi submetido ao Comitê de Ética em Pesquisa (CEP) da UNINORTE e aprovado com número CAAE: 40847620.7.0000.8028 e Parecer Consubstanciado n.ำ 4.480.074 de 21 de dezembro de 2020.

\section{RESULTADOS E DISCUSSÃO}

A amostra final foi composta por vinte e dois acadêmicos de enfermagem com predominância de acadêmicos do décimo período e do sexo feminino. O Quadro 1 registra a caracterização dos participantes do estudo.

Quadro 1 - Caracterização dos acadêmicos de Enfermagem participantes do estudo.

\begin{tabular}{|c|c|c|c|}
\hline ACADÊMICOS & SEXO & PERÍODO & IDADE \\
\hline $\mathrm{A} 1$ & $\mathrm{~F}$ & $8^{\circ}$ & 22 \\
\hline $\mathrm{A} 2$ & $\mathrm{~F}$ & $8^{\circ}$ & 32 \\
\hline A3 & $\mathrm{F}$ & 90 & 29 \\
\hline A4 & $\mathrm{F}$ & $9^{\circ}$ & 22 \\
\hline A5 & $\mathrm{F}$ & 9응 & 31 \\
\hline A6 & $\mathrm{F}$ & $10^{\circ}$ & 34 \\
\hline A7 & $\mathrm{F}$ & $10^{\circ}$ & 44 \\
\hline A8 & $\mathrm{F}$ & $10^{\circ}$ & 31 \\
\hline A9 & $\mathrm{F}$ & $10^{\circ}$ & 23 \\
\hline A10 & $\mathrm{F}$ & $10^{\circ}$ & 32 \\
\hline A11 & $\mathrm{F}$ & $10^{\circ}$ & 31 \\
\hline $\mathrm{A} 12$ & $\mathrm{~F}$ & $10^{\circ}$ & 34 \\
\hline A13 & $\mathrm{F}$ & $10^{\circ}$ & 46 \\
\hline A14 & $\mathrm{F}$ & $10^{\circ}$ & 36 \\
\hline A15 & $\mathrm{F}$ & $10^{\circ}$ & 23 \\
\hline A16 & $\mathrm{F}$ & $10^{\circ}$ & 38 \\
\hline A17 & $\mathrm{F}$ & $10^{\circ}$ & 46 \\
\hline A18 & $\mathrm{F}$ & $10^{\circ}$ & 40 \\
\hline A19 & $\mathrm{F}$ & $10^{\circ}$ & 41 \\
\hline A 20 & $\mathrm{~F}$ & $10^{\circ}$ & 41 \\
\hline A21 & $\mathrm{F}$ & $10^{\circ}$ & 41 \\
\hline A22 & $M$ & $10^{\circ}$ & 44 \\
\hline
\end{tabular}

Fonte: Fernandes ECC, et al., 2021. 
A enfermagem é uma profissão que em sua grande maioria é do sexo feminino, o que denota a tradição de que ainda há uma feminilização da profissão (MACHADO DF, 2020). Segundo pesquisa realizada pelo Conselho Federal de Enfermagem (COFEN) em 2020, existem cerca de 2.502.291 profissionais de enfermagem atuantes em todo o Brasil, destes, 32,0\% (do total de 615.712) representam a categoria de enfermeiros, os quais $87,3 \%$ correspondem ao sexo feminino e $12,7 \%$ ao sexo masculino, ratificando predominância da enfermagem composta por mulheres em todo o país (SILVA VG e RIBEIRO PM, 2020).

A análise do conteúdo foi identificada como parte fundamental do processo de investigação como estratégia de compreensão, a partir do olhar dos acadêmicos de enfermagem. Das respostas analisadas emergiram três categorias temáticas: Práticas educativas implementadas no cenário acadêmico; Temáticas de discussão sobre violência contra a mulher e; O futuro profissional de enfermagem e a violência contra a mulher.

\section{Práticas educativas implementadas no cenário acadêmico}

Em consequência do aumento agravante e da considerável potencial de destruição, a violência contra as mulheres tem recebido cada vez mais atenção nos âmbitos acadêmicos. Neste sentido, diferentes disciplinas de diversas instituições universitárias têm desenvolvido projetos de pesquisa e de intervenção considerados fenômenos sociais complexos, multifacetados e históricos que afetam indivíduos, famílias, comunidades e a sociedade em nível sistemático e holístico (MOURA RCM, et al., 2018).

Para isso, é necessário que instituições do ensino em especial no curso de enfermagem revejam as estruturas curriculares de seus cursos, a fim de coloborar com o compartilhamento de conhecimento sobre as questões de gênero e discutir o tema da violência contra mulheres e outras minorias vulneráveis (FERRAZ CS e SOUZA FB, 2018).

No que tange as práticas educativas implementadas sobre violência contra mulher no cenário acadêmico, alguns participantes relataram que uso de metodologias mais ativas como rodas de conversa, projetos aplicativos, trabalhos em equipe e simulação realística são estratégicas:

"Rodas de conversa, pois permite uma reflexão sobre o assunto abordado" (A6).

"Trabalhos em equipe com apresentação e simulação realística, rodas de conversa, projetos aplicativos e até mesmo Trabalho de Conclusão de Curso (TCC), com o intuído de alertar e conscientizar a todos que não se deve aceitar a violência" (A18).

A utilização de métodos ativos como ferramenta de estudo para o ensino de graduação em enfermagem, a fim de capacitar os discentes a prognosticar a realidade das situações no quesito da prática profissional e prepará-los para novas técnicas de resolução para problemáticas comuns de saúde no exercício do enfermeiro (VEIGA AM, 2020).

O uso de metodologias ativas no ensino da enfermagem, constitui um novo paradigma para a formação de enfermeiros críticos e reflexivos, trazendo maior aproximação da realidade no percurso da graduação, nesse contexto, a principal vantagem do uso de medotologias ativas de ensino em sala de aula é estimular o desenvolvimento de cada acadêmico como pessoa, cidadão e profissional (CLAPIS MJ, et al, 2018).

O depoimento do participante A6 corrobora com a descrição da literatura, pois por meio das rodas de conversa, é possível a experiência dos acadêmicos de enfermagem ganhar um novo significado e promover a reflexão sobre a temática discutida. Moreira GAR, et al. (2018) frisa que existe uma grande importância a respeito das rodas de conversas, das apresentações de trabalhos em equipe e projetos aplicativos relacionados à violência contra a mulher, pois são estratégias educacionais que permite maior visibilidade do assunto, colaborando para que os acadêmicos se expressem sobre o tema (BURAFALDI LA, et al., 2017).

Em complemento, Sampaio LJ, et al. (2018) referem que as rodas de conversa possibilitam encontros dialógicos criando assim possibilidades de produção e ressignificação de sentidos e saberes sobre a 
experiência de cada acadêmico de enfermagem sobre a temática da VCM. Para Sobrinho NC, et al. (2019) e Martins FL, et al. (2019), as rodas de conversa produzem conhecimentos coletivos e contextualizados já que são construídas pela fala crítica e escuta sensível de forma lúdica favorecendo o entrosamento e a confiança entre os acadêmicos.

Sobre o uso dos projetos aplicativos, Machado DF (2020) referem que essa estratégia metodológica de ensino aprendizagem buscam sensibilizar e mobilizar os acadêmicos de enfermagem a refletir acerca do tema abordado e com seu projeto de vida. Em estudo realizado por Sobrinho NC, et al. (2019) em uma IES, os acadêmicos de enfermagem evidenciaram que o trabalho em equipe com apresentação de uma simulação realística sobre a violência contra mulher, é de grande relevância para o conhecimento e aprendizado dos participantes.

De acordo com Melo ALJM e Vieira LSAV (2017) os acadêmicos de enfermagem apontam também, que as palestras e aulas sobre a temática da VCM contribuem para a sua formação profissional, e principalmente, para o seu conhecimento científico, e nesta categoria temática emergiram como outras práticas educativas, as palestras e aulas teóricas que abordam a temática, conforme as falas mencionadas:

"Palestras e aulas debatendo o assunto da violência contra mulher" (A19).

"Palestras e aulas sobre o assunto da VCM" (A7).

"Palestras sobre a violência contra mulher" (A14).

Em se tratando do momento em que a temática foi abordada, a maioria dos acadêmicos de enfermagem revelam que foi na disciplina de Assistência Integral à Saúde da Mulher ou disciplina similar, que o ensino sobre violência foi abordado, e quando abordaram esse tema, foi de maneira superficial, o que determinará futuramente que 0 acadêmico busque futuramente soluções que minimizassem as lacunas de seu aprendizado sobre o fenômeno, como atualizações, aperfeiçoamentos e especializações (RODRIGUES JBS, et al., 2021).

Peralva TRP, et al. (2016) apontam que faz-se necessário a intensificação de palestras educativas com foco para a atuação e assistência de enfermagem à mulher que sofre violência, pois, com isso amplia-se espaços de reflexão e apredizado para este tema de grande relevância no cenário atual por tratar-se de uma problemática de saúde pública.

Queiroz RAQ e Cunha TARC (2018) evidenciam em seu estudo que palestras e seminários mais aprofundados sobre essa temática são fundamentais para caracterizar e evidenciar as formas de violência, principais motivos, causadores da violência, bem como as formas como essas mulheres são atendidas nos serviços de saúde, estimulando principalmente a produção científica que a enfermagem vem produzindo nos últimos anos.

\section{Temáticas de discussão sobre violência contra a mulher}

O contexto da violência contra a mulher no curso de enfermagem possibilita maior aprendizado, além de promover pensamento crítico e postura ética aos acadêmicos, colabora para o desenvolvimento de evidências científicas a respeito da temática (SOUZA CND, et al., 2019).

Silva VG e Ribeiro PM (2020), destacam que a violência obstétrica é um dos assuntos mais abordados dentro das disciplinas que se referem à saúde da mulher durante a graduação de enfermagem. As falas dos participantes do estudo também referem a violência obstétrica como uma das temáticas mais discutidas entre os acadêmicos de enfermagem:

\section{"Violência obstétrica, é algo que ainda ocorre nas maternidades" (A11).}

"A Violência obstétrica é um tipo de violência contra mulher" (A4).

De acordo com estudo estudo realizado por Leal SYP (2018), a violência obstétrica é algo que ainda ocorre nas maternidades e que não deveria acontecer devido os avanços tecnológicos que existem e das informações que precisam ser repassadas tanto para as mulheres, famílias e para os profissionais. 
Corroborando, o estudo realizado por Matoso LML (2018) na busca da compreensão do olhar do acadêmico de enfermagem, buscou-se também a compreensão dos mesmos sobre violência obstétrica, para eles, é um tipo de agressão específica praticada em mulheres no ciclo gravídico puerperal, podendo causar danos mais sérios à saúde da mulher.

O conhecimento dos acadêmicos de enfermagem sobre o contexto da violência obstétrica, possibilita maior entendimento sobre o assunto (GALVÃO, RL, et al., 2021). Outras temáticas discutidas na academia sobre violência contra a mulher descritas pelos acadêmicos de enfermagem foram 0 acolhimento e a abordagem a vítima, conforme os relatos a seguir:

"Acolhimento e abordagem" (A8).

"Abordagem a vítima de violência" (A7).

Acolhimento das mulheres vítimas de violência" (A18).

Lemes MF, et al. (2015), destacam que é importante que os acadêmicos de enfermagem saibam como acolher, abordar, e realizar uma assistência de enfermagem às mulheres vitímas de violência como preconizado pelo Ministério da Saúde, visto que na grande maioria das vezes, esse tipo de assunto é pouco abordado durante a formação acadêmica.

O acolhimento é de extrema importância para a qualidade e humanização da atenção, pode-se dizer que o acolhimento é um conjunto de medidas, posturas e atitudes dos profissionais de saúde, que visam garantir credibilidade e consideração à situação de VCM (SANTOS MS, et al., 2021).

O enfermeiro desempenha um papel relevante na assistência à mulher vítima de violência, pois exerce justamente no acolhimento e cuidado dessas pacientes, com o objetivo de promover condições favoráveis para a recuperação da saúde em caso de extrema agressividade sem diferenciar os níveis de violência. (RODRIGUES FA, et al., 2017). Além disso, os acadêmicos de enfermagem presentes devem saber como agir em caso de agressão à mulher para que possam dar apoio e compreensão (JARDIM DMB e MODENA CM, 2018).

Outra temática discutida diz respeito à perspectiva das redes de apoio social a mulher vítima de alguma forma de agressão. A mulher que recebe o suporte da sua rede de apoio social sente-se valorizada, cuidada e apoiada por alguém que está afetivamente disponível:

"Rede de apoio as mulheres vítimas de violência" (A20).

Barbosa LC, et al. (2017) sugerem que as ações de enfermagem sejam elencadas através de ações em rede ao apoio à mulher vítima de violência e, não simplesmente no setor da saúde, uma vez que este é um agravo que extrapola esta área, perpassando por âmbitos sociais e culturais. Xavier AAP e Silva EG (2019) afirmam que além de existir as políticas públicas de saúde e as redes de apoio para medidas a serem tomadas pelos profissionais de saúde, o profissional de enfermagem é mencionado como peça fundamental no cuidado de mulheres em situação de violência, por ser o profissional que tem contato mais direto com a vítima.

\section{O futuro profissional de enfermagem e a violência contra a mulher}

Saldanha MPAl e Gonçalves HC (2016) enfatizam ainda que em algum momento durante a trajetória, o futuro profissional enfermeiro pode vir a se defrontar com essa situação, exigindo conhecimento específico e habilidade para que assim possa realizar uma asssitência difereciada a essa mulher, além de também estar atuando na promoção à saúde, o qual são confirmadas com as falas dos participantes abaixo quando afirmam além de conhecimento específico, a necessidade de uma assistência diferenciada:

"A importância é essencial pois nós devemos estar preparados para esse tipo de cuidado por se tratar de uma assistência diferenciada que requer uma atenção necessária e precisa" (A22).

"Ter conhecimento específico no que diz respeito a assistência de enfermagem prestada às pacientes" (A13). 
O exposto evidencia a necessidade do profissional de enfermagem ter um olhar diferenciado quando se trata de um caso de violência contra mulher e a qualidade da sua assistência. Os futuros enfermeiros devem estar preparados para prestar uma assistência diferenciada que contenham ações de planejamento e mecanismo bem definidos que atendam às necessidades individuais às vítimas (SANTOS MS, et al. 2021). A eficiência dessa assistência requer uma atenção necessária e precisa que colabora para que os cuidados de saúde sejam prestados contribuindo para minimizar o impacto causado na vida dessas mulheres, servindo de instrumento para um excelente atendimento, fortalecendo-as para enfrentar possíveis traumas (OLIVEIRA TR, et al., 2017).

A temática VCM deve estar presente em todos semestres, integrar outras disciplinas e ter relação com a prática assistencial que permita o aprendizado a partir da relação da realidade vivida pelos acadêmicos e às questões da vida das pessoas (SILVA EB, et al., 2015). As IES principalmente as da esfera da saúde, precisam ter como foco a formação de especialistas que adquiriam competências e capacidades a partir da produção do conhecimento. Neste sentido, os participantes ratificam suas falas abaixo:

"Nas instituições superiores principalmente no curso de enfermagem, devem abordar o assunto da VCM" (A3).

"As instituições repensem o modo de abordar a temática sobre a VCM no curso de enfermagem" (A8).

"Dificuldade do profissional para a identificação dos casos de VCM" (A12).

Xavier AAP e Silva EG (2019) destacam que as IES tem formado profissionais que apresentam fragilidades na identificação e atendimento das vítimas e apontaram a necessidade de preencher lacunas na formação de professores. Os docentes devem utilizar métodos inovadores para estimular os acadêmicos a desenvolver uma consciência crítica sobre as questões que afetam a vida e a saúde da população, além de incentivá-los a reconhecer seu importante papel como membros da sociedade contra a violência.

Entende-se que a própria academia propicia a aprendizagem fragmentada, deixa de trabalhar dentro dos limites das disciplinas e a teoria e a prática ficam fora de alcance, o que acaba não propiciando a reflexão e crítica dos futuros profissionais (SOUZA CND, et al., 2019).

Os estudantes do último ano dos cursos de graduação em enfermagem, medicina e odontologia da Universidade Federal de Santa Catarina, evidenciaram que é difícil para os profissionais de saúde identificarem casos de violência devido à falta de sensibilidade e escuta durante o atendimento (OLIVEIRA TR, et al., 2017).

Barbosa LC, et al. 2017) em sua pesquisa realizada em uma IES indicaram que é necessário que as instituições repensem a forma como a academia resolve esses problemas, proporcionando aos alunos um espaço prático, deixando que os mesmos entrem em contato com usuários reais e saibam reconhecer e atuar, usando a audição como forma de orientação para o trabalho em equipe multidisciplinar. Moura RCM, et al. (2018), enfatizam que as IES devem reavaliar suas estruturas curriculares e planos de ensino para sanar o problema.

A integração ensino-serviço é essencial para fortalecer a política de educação permanente em saúde, por meio da atuação das IES e dos profissionais das redes de atenção à saúde, na supervisão acadêmica das atividades desempenhadas pelos acadêmicos, o qual são confirmadas com a fala do participante abaixo:

\section{"Estágio e condutas específicas na VCM" (A20).}

Nos estágios curriculares as discussões permanentes sobre a temática são quase inexistentes, essas práticas são comumente previstas em campanhas ou datas alusivas. O estágio é uma etapa indispensável na formação acadêmica do enfermeiro, sendo desempenhado na APS, em hospitais e ambulatórios e em centros de atenção primária. Nessas circunstâncias, o discente aperfeiçoa seu ofício profissional, vinculando 
a teoria à prática, outrossim, os acadêmicos de enfermagem são configurados como promotores da inovação local, em virtude de utilizarem os conhecimentos mais recentes, o que tem produzido uma resposta positiva nas suas ações e intervenções (CLAPIS MJ, et al., 2018).

Com o término desde a graduação, os discentes de enfermagem subsistem momentos de aprendizagem associados na teórica e prática, vivenciando etapas de investigação, detalhamento de cena e demonstração, e após a entrada no estágio sucederão em responder rapidamente ao estímulo da cena prática realizando tarefas, incluindo em suas funções hodiernamente adquiridas (ARAÚJO VABT, et al., 2016).

Dessa forma, o estágio proporciona uma integração entre o meio acadêmico e profissional, aproximando os discentes da realidade e oferece oportunidades de mobilização efetiva de conhecimentos, habilidades e atitudes aprendidas durante o ciclo formativo. Sua finalidade é proporcionar ao acadêmico articular teoria e prática, por meio de estratégias que ampliem habilidades de comunicação, flexibilidade e tomada de decisão (CLAPIS MJ, et al., 2018).

\section{CONCLUSÃO}

Compreende-se que a violência contra mulher é uma temática que necessita de maior aprofundamento e abordagem ainda na graduação em enfermagem, com uso de metodologias ativas que potencializem de maneira crítica e problematizadora as habilidades e competências do futuro profissional. Além disso, deve estar contido nos planos de ensino acadêmico, estratégias que fortaleçam a integração ensino e serviço. Deste modo, estabelece à importância da realização de novos estudos voltados para abordagens que relacionem formação acadêmica e prática profissional em enfermagem a fim de contribuir com uma assistência humanizada, protetiva e eficaz.

\section{REFERÊNCIAS}

1. ARAÚJO VABT, et al. Formação e práticas de docentes de um curso de graduação em enfermagem. Ciência Saúde Coletiva, 2016; 38(1): 69-79.

2. BARBOSA LC, et al. Violência obstétrica: revisão integrativa de pesquisas qualitativas. Revista Brasileira de Enfermagem, 2017; 35(2): 190-207.

3. BURAFALDI LA, et al. Violência de gênero: comparação da mortalidade por agressão em mulheres com e sem notificação prévia de violência. Ciência Saúde Coletiva, 2017; 22(9): 2929-2938.

4. BRASIL. Ministério da Educação. Resolução CNE/CES no 3, de 07 de novembro de 2001: diretrizes curriculares nacionais do curso de graduação em enfermagem. Brasília, 2016. Disponível em:http://www.cofen.gov.br/resoluocne-ces-n-3-de-7-de-novembro-de-2001-diretrizes-nacionais-curso-graduaao-enfermagem_6933.html. Acessado em: 16 de maio de 2021.

5. CLAPIS MJ, et al. Metodologia da problematização no ensino da atenção básica à saúde. Revista Brasileira de Enfermagem, 2018; 1(2): 12-20.

6. DELZIOVO CR, et al. Violência sexual contra a mulher e o atendimento no setor saúde em Santa Catarina - Brasil. Ciência Saúde Coletiva, 2018; 1(2): 20-24.

7. FERRAZ CS, SOUZA FB. Violência doméstica contra a mulher: um olhar da gestaltterapia. Revista. Conexão Eletrônica, 2018; 15(1): 20-30.

8. GALVÃO RL, et al. Atuação dos profissionais de enfermagem frente às mulheres vítimas de violência doméstica. Revista Eletrônica Acervo Saúde, 2021; 13(1).

9. JARDIM DMB, MODENA CM. A violência obstétrica no cotidiano assistencial e suas características. Rev. Latino-Am. Enfermagem, 2018; 26(1): 30-35.

10. LEAL SYP. Percepção de enfermeiras obstétricas acerca da violência obstétrica. Cogitare Enferm, 2018; 23(2): 1218.

11. LEMES MF, et al. Representações sociais em mulheres em contexto de violência doméstica. Perspectivas em Psicologia, 2015; 10(2): 5-10.

12. MARTINS FL, et al. Violência Obstétrica: Uma expressão nova para um problema histórico. Revista Saúde em Foco, 2019; 11(2): 413-423.

13. MATOSO LML. O papel do enfermeiro frente à violência obstétrica. Revista Eletrônica da FAINOR, 2018; 11(1): 4965.

14. MACHADO DF. Violência contra a mulher: o que acontece quando a Delegacia de Defesa da Mulher está fechada? Ciência Saúde coletiva, 2020; 25(2).

15. MELO ALJM, et al. A percepção de mulheres sobre violência de gênero e sexualidade feminina. Rev. Min. Enfermagem. REME, 2017; 1(1): 1-8. 
16. MOURA RCM, et al. Cuidados de enfermagem na prevenção da violência obstétrica. Enfermagem em Foco, 2018; 9(4): 60-65.

17. MOREIRA GAR, et al. Qualificação de profissionais da saúde para a atenção ás mulheres em situação de violência sexual. Revista Trabalho e Educação em Saúde, 2018; 3(16): 1039-1055.

18. ORGANIZAÇÃO MUNDIAL DE SAÚDE. OMS. Relatório mundial sobre violência e saúde. Genebra, 2018. Disponível em: https://www.who.int/eportuguese/publications/pt/. Acessado em: 03 de abril de 2021.

19. OLIVEIRA FS, et al. Violência doméstica e sexual contra a mulher: revisão integrativa. Ciência Saúde Coletiva, 2017; 8(1): 275-284

20. PERALVA TRP, et al. Violência doméstica na percepção de enfermeiros de serviço de emergência. Revista de ciências e tecnologia, 2016;2(3).

21. QUEIROZ RAQ, CUNHA TARC. A violência psicológica sofrida pelas mulheres: invisibilidade e memória. Rev. Latino-Am. Enfermagem, 2018; 20(10): 86-95.

22. RODRIGUES FA, et al. Violência obstétrica no processo de parturição em maternidades vinculadas à Rede Cegonha. Revista Gaúcha Enfermagem, 2017; 32(2): 78-84.

23. RODRIGUES JBS, et al. Atuação do enfermeiro frente a mulher vítima de violência sexual. Revista Eletrônica Acervo Saúde, 2021; 13(2).

24. ROSÁRIO AC, et al. Violência entre pares na adolescência: Um estudo com estudantes no início e no final do 3. ำ ciclo do ensino básico. Revista de Psicologia, 2017; 31(2): 57-67.

25. SAMPAIO LJ, et al. Violência doméstica: caracterização das mulheres vítimas de agressão física em Joinville. Revista Recien, 2020; 1(10).

26. SALDANHA MPAI, GONÇALVES HS. Práticas de empoderamento feminino na América Latina. Revista Enfermagem Contemporânea, 2016; 2(1): 16-26.

27. SANTOS AG, et al. Domínios dos transtornos mentais comuns em mulheres que relatam violência por parceiros íntimos. Revista Latina Americana, 2015; 26(4).

28. SANTOS MS, et al. A relevância da enfermagem a mulher vítima de violência doméstica. Revista Eletrônica Acervo Saúde, 2021; 13(4).

29. SILVA VG, RIBEIRO PM. Violência contra as mulheres na prática de enfermeiras da atenção primária à saúde. Esc. Anna Nery 2020; 24(4).

30. SOBRINHO NC et al. Violência contra a mulher: a percepção dos graduandos de enfermagem. Revista Latino Americana de Enfermagem, 2019; 1(1): 120-130.

31. SOUZA CND, et al. O papel da enfermagem na violência sexual contra a mulher. Revista Brasileira Interdisciplinar de Saúde, 2019; 4(1): 31-36.

32. TEIXEIRA MCB, et al. Entre espelhos: a formação em saúde e sua produção de violência. Revista Eletrônica de Enfermagem, 2018; 18(2): 156-165.

33. VEIGA AM. Uma virada epistêmica feminista (negra): conceitos e debates. Tempo e Argumento, 2020; 12(9).

34. XAVIER AAP, SILVA EG. Assistência de enfermagem no atendimento de mulheres em situação de violência na atenção básica. Rev Inic Cient Ext, 2019; 2(2): 293-300. 